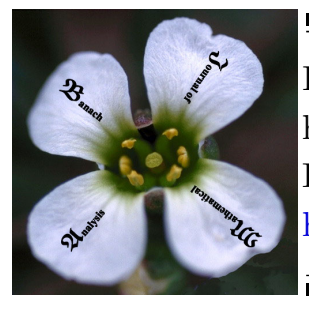

Banach J. Math. Anal. 9 (2015), no. 2, 21-34

http://doi.org/10.15352/bjma/09-2-3

ISSN: $1735-8787$ (electronic)

http://projecteuclid.org/bjma

\title{
HARDY-TYPE INEQUALITIES ON THE WEIGHTED CONES OF QUASI-CONCAVE FUNCTIONS
}

\author{
L.-E. PERSSON ${ }^{1 *}$, G. E. SHAMBILOVA ${ }^{2}$ AND V. D. STEPANOV ${ }^{3}$ \\ Communicated by L. Castro
}

\begin{abstract}
The complete characterization of the Hardy-type $L^{p}-L^{q}$ inequalities on the weighted cones of quasi-concave functions for all $0<p, q<\infty$ is given.
\end{abstract}

\section{INTRODUCTION}

Let $\mathbb{R}_{+}:=[0, \infty)$. Denote $\mathfrak{M}$ the set of all measurable functions on $\mathbb{R}_{+}, \mathfrak{M}^{+} \subset$ $\mathfrak{M}$ the subset of all non-negative functions and $\mathfrak{M}^{\downarrow} \subset \mathfrak{M}^{+}\left(\mathfrak{M}^{\uparrow} \subset \mathfrak{M}^{+}\right)$is the cone of all non-increasing (non-decreasing) functions. Let $\varphi \in \mathfrak{M}^{+}$be a smooth strictly increasing function such that $\varphi(0)=0, \varphi(\infty)=\infty$. Then we say, that $\varphi$ is admissible. The cone of $\varphi$-quasi-concave functions is defined by

$$
\Omega_{\varphi}:=\left\{f \in \mathfrak{M}^{\uparrow}: \frac{f}{\varphi} \in \mathfrak{M}^{\downarrow}\right\} .
$$

Numerous papers were recently devoted to analysis of classical operators on the weighted cones of quasi-monotone and quasi-concave functions (see, for instance, recent survey [7], the paper [11], and literature given there). In particular, it plays an important role in the Lorentz spaces (see [1], [13], [3]).

Date: Received: Mar. 8, 2014; Accepted: Jun. 9, 2014.

* Corresponding author.

2010 Mathematics Subject Classification. Primary 26D15; Secondary 47G10.

Key words and phrases. Hardy-type inequality, weight, measure, Lorentz space, concave function, quasi-concave function. 
Let $u, v, w \in \mathfrak{M}^{+}, 0<p, q<\infty$. One of the challenging problems is to characterize the inequalities

$$
\left(\int_{0}^{\infty}[A f(x)]^{q} u(x) d x\right)^{\frac{1}{q}} \leq C_{A}\left(\int_{0}^{\infty}[f(x)]^{p} v(x) d x\right)^{\frac{1}{p}}, f \in \Omega_{\varphi}
$$

and

$$
\left(\int_{0}^{\infty}[B f(x)]^{q} u(x) d x\right)^{\frac{1}{q}} \leq C_{B}\left(\int_{0}^{\infty}[f(x)]^{p} v(x) d x\right)^{\frac{1}{p}}, f \in \Omega_{\varphi},
$$

where

$$
A f(x):=\int_{0}^{x} f(y) w(y) d y
$$

and

$$
B f(x):=\int_{x}^{\infty} f(y) w(y) d y
$$

In a perfect form characterization of (1.1) and (1.2) means sharp two-sided estimates of the (least possible) constants $C_{A}$ and $C_{B}$, respectively, by integral functionals depending on weights and parameters of summation, so that finitness of the functional implies the validity of the inequality and vice versa. Also, this problem is closely related to the boundedness of the Hardy-Littlewood maximal operator in weighted Lorentz $\Gamma$-spaces and during last two decades it was solved for all parameters $0<p, q<\infty$ except $0<q<1$ (see [4], [14],[11]). In the present paper we fill up the gap. Moreover, we use alternative method, which brings explicit criteria rather different from [14] and [11] and from implicit results of [4] and [5].

Section 2 is devoted to preliminaries. The main results are given in the Section 3.

We use signs $:=$ and $=$ : for determining new quantities and $\mathbb{Z}$ for the set of all integers. For positive functionals $F$ and $G$ we write $F \lesssim G$, if $F \leq c G$ with some positive constant $c$, which depends only on irrelevant parameters. $F \approx G$ means $F \lesssim G \lesssim F$ or $F=c G$. $\chi_{E}$ denotes the characteristic function (indicator) of a set $E$. Uncertainties of the form $0 \cdot \infty, \frac{\infty}{\infty}$ and $\frac{0}{0}$ are taken to be zero. $\square$ stands for the end of a proof.

\section{Preliminaries}

Denote

$$
\Omega_{0, p}:=\left\{f \in \mathfrak{M}^{\uparrow}: \frac{f(t)}{t^{p}} \in \mathfrak{M}^{\downarrow}\right\}, 0<p<\infty .
$$

It is well known ([2], Proposition 2.5.10), that for any $f \in \Omega_{0,1}$ there is a concave function $\tilde{f}$ such that

$$
\frac{1}{2} \tilde{f} \leq f \leq \tilde{f}
$$


and it was shown in ([14], Lemma 2.3), that for every concave function there exists a sequence $\left\{h_{n}\right\} \in \mathfrak{M}^{+}$such that

$$
g_{n}(x):=\int_{0}^{x} d y \int_{y}^{\infty} \frac{h_{n}(s) d s}{s} \uparrow \tilde{f}(x) .
$$

Also, observe that

$$
f \in \Omega_{\varphi} \Leftrightarrow f\left(\varphi^{-1}\right) \in \Omega_{0,1}
$$

and for $0<p<\infty$

$$
f \in \Omega_{\varphi} \Leftrightarrow f^{p} \in \Omega_{\varphi^{p}}
$$

Thus, repeating the argument of ([14], Lemma 2.3) we obtain the following.

Lemma 2.1. Let $0<p<\infty$ and $\varphi$ is admissible. Then for any $f \in \Omega_{\varphi}$ there are $\tilde{f}(x) \approx[f(x)]^{p}$ and a sequence $\left\{h_{n}\right\} \in \mathfrak{M}^{+}$such that

$$
g_{n}(x):=\int_{0}^{\varphi^{p}(x)} d y \int_{y}^{\infty} \frac{h_{n}(s) d s}{s} \uparrow \tilde{f}(x) .
$$

If $0<p<\infty$ and $v \in \mathfrak{M}^{+}$we define

$$
L_{v}^{p}:=\left\{h \in \mathfrak{M}:\|h\|_{L_{v}^{p}}:=\left(\int_{0}^{\infty}|h(x)|^{p} v(x) d x\right)^{\frac{1}{p}}<\infty\right\}
$$

and we write $L^{p}$ when $v \equiv 1$. Let $0<q<\infty$ and $w \in \mathfrak{M}^{+}$. Consider operators $T$ and $S$ of the form

$$
T h(x):=\left(\int_{0}^{x} w(y)\left(\int_{y}^{\infty} h\right)^{q} d y\right)^{\frac{1}{q}}
$$

and

$$
\operatorname{Sh}(x)=\left(\int_{0}^{x} w(y)\left(\int_{0}^{y} h\right)^{q} d y\right)^{\frac{1}{q}} .
$$

Let $u, v, w \in \mathfrak{M}^{+}$be weights, $1 \leq p<\infty, 0<r<\infty$. The following inequalities

$$
\|T h\|_{L_{u}^{r}} \leq C_{T}\|h\|_{L_{v}^{p}}, h \in \mathfrak{M}^{+}
$$

and

$$
\|S h\|_{L_{u}^{r}} \leq C_{S}\|h\|_{L_{v}^{p}}, h \in \mathfrak{M}^{+}
$$

have been characterized in [8] and [9] for the operator $T$ and in [12] for the both. The criteria in [8] and [9] were found by the discretization method [6] in a more complicated form than in [12]. Below we give the related results from [12] in a slightly improved form.

We suppose for simplicity that $0<\int_{t}^{\infty} u<\infty$ for all $t>0$ and define the functions $\zeta:[0, \infty) \rightarrow[0, \infty), \zeta^{-1}:[0, \infty) \rightarrow[0, \infty)$ by

$$
\begin{gathered}
\zeta(x):=\sup \left\{y>0: \int_{y}^{\infty} u \geq \frac{1}{2} \int_{x}^{\infty} u\right\}, \\
\zeta^{-1}(x):=\sup \left\{y>0: \int_{y}^{\infty} u \geq 2 \int_{x}^{\infty} u\right\} .
\end{gathered}
$$


Here $\sup \varnothing=0$. Let $\zeta^{-2}:=\zeta^{-1}\left(\zeta^{-1}\right)$. For $0 \leq c<d<\infty$ and $h \in \mathfrak{M}^{+}$we put

$$
\begin{aligned}
\left(H_{c, d} f\right)(x) & :=\chi_{(c, d]}(x) \int_{x}^{\zeta(d)} h, \\
\left(H_{d} f\right)(x) & :=\chi_{(0, d]}(x) \int_{x}^{\infty} h, \\
\left(H_{c, d}^{*} f\right)(x) & :=\chi_{(c, d]}(x) \int_{\zeta^{-1}(c)}^{x} h, \\
\left(H_{d}^{*} f\right)(x) & :=\chi_{(0, d]}(x) \int_{0}^{x} h .
\end{aligned}
$$

Theorem 2.2. ([12], Theorems 4 and 5.) Let $1 \leq p<\infty, 0<r<\infty, 0<q<\infty$ and $\frac{1}{s}:=\frac{1}{r}-\frac{1}{p}$ for $r<p$.

(a) For validity of the inequality (2.1) it is necessary and sufficient that the inequality

$$
\left(\int_{0}^{\infty} u(x)\left(\int_{0}^{x} w\right)^{\frac{r}{q}}\left(\int_{x}^{\infty} h\right)^{r} d x\right)^{\frac{1}{r}} \leq A_{0}\|h\|_{L_{v}^{p}}
$$

holds for all $h \in \mathfrak{M}^{+}$and the constant

$$
A_{1}:= \begin{cases}\sup _{t \in(0, \infty)}\left(\int_{t}^{\infty} u\right)^{\frac{1}{r}}\left\|H_{t}\right\|_{L_{v}^{p} \rightarrow L_{w}^{q},} & p \leq r \\ \left(\int_{0}^{\infty} u(x)\left(\int_{x}^{\infty} u\right)^{\frac{s}{p}}\left\|H_{\zeta^{-1}(x), \zeta(x)}\right\|_{L_{v}^{p} \rightarrow L_{w}^{q}}^{s} d x\right)^{\frac{1}{s}}, & r<p\end{cases}
$$

is finite. Moreover, $C_{T} \approx A_{0}+A_{1}$.

(b) The inequality (2.2) is true if and only if the inequality

$$
\left(\int_{0}^{\infty} u(x)\left(\int_{\zeta^{-2}(x)}^{x} w\right)^{\frac{r}{q}}\left(\int_{0}^{\zeta^{-2}(x)} h\right)^{r} d x\right)^{\frac{1}{r}} \leq \boldsymbol{A}_{0}\|h\|_{L_{v}^{p}}
$$

holds for all $h \in \mathfrak{M}^{+}$and the constant

$$
\boldsymbol{A}_{1}:= \begin{cases}\sup _{t \in(0, \infty)}\left(\int_{t}^{\infty} u\right)^{\frac{1}{r}}\left\|H_{t}^{*}\right\|_{L_{v}^{p} \rightarrow L_{w}^{q},} & p \leq r \\ \left(\int_{0}^{\infty} u(x)\left(\int_{x}^{\infty} u\right)^{\frac{s}{p}}\left\|H_{\zeta^{-1}(x), \zeta(x)}^{*}\right\|_{L_{v}^{p} \rightarrow L_{w}^{q}}^{s} d x\right)^{\frac{1}{s}}, & r<p\end{cases}
$$

is finite. Moreover, $C_{S} \approx \boldsymbol{A}_{0}+\boldsymbol{A}_{1}$.

Analogously, to solve the inequality (1.2) we need sublinear positive operators $\mathcal{T}$ and $\mathcal{S}$ of the form

$$
\mathcal{T} h(x):=\left(\int_{x}^{\infty} w(t)\left(\int_{0}^{t} h\right)^{q} d t\right)^{\frac{1}{q}}, h \in \mathfrak{M}^{+}
$$




$$
\mathcal{S} h(x):=\left(\int_{x}^{\infty} w(t)\left(\int_{t}^{\infty} h\right)^{q} d t\right)^{\frac{1}{q}}, h \in \mathfrak{M}^{+}
$$

and weighted inequalities

$$
\begin{gathered}
\|\mathcal{T} h\|_{L_{u}^{r}} \leq C_{\mathcal{T}}\|h\|_{L_{v}^{p}}, h \in \mathfrak{M}^{+}, \\
\|\mathcal{S} h\|_{L_{u}^{r}} \leq C_{\mathcal{S}}\|h\|_{L_{v}^{p}}, h \in \mathfrak{M}^{+},
\end{gathered}
$$

where $u, v \in \mathfrak{M}^{+}, 1 \leq p<\infty, 0<r<\infty$, which have been characterized in [12]. Below we give the related results from [12].

We suppose for simplicity that $0<\int_{0}^{t} u<\infty$ for all $t>0$ and define the functions $\sigma:[0 ; \infty) \rightarrow[0 ; \infty), \sigma^{-1}:[0 ; \infty) \rightarrow[0 ; \infty)$, by

$$
\begin{aligned}
\sigma(x) & :=\inf \left\{y>0: \int_{0}^{y} u \geq 2 \int_{0}^{x} u\right\}, \\
\sigma^{-1}(x) & :=\inf \left\{y>0: \int_{0}^{y} u \geq \frac{1}{2} \int_{0}^{x} u\right\} .
\end{aligned}
$$

Let $\sigma^{2}:=\sigma(\sigma)$. For $0 \leq c<d<\infty$ and $h \in \mathfrak{M}^{+}$we put

$$
\begin{aligned}
\mathcal{H}_{c} h(x) & :=\chi_{[c, \infty)}(x) \int_{0}^{x} h, \\
\mathcal{H}_{c, d} h(x) & :=\chi_{[c, d)}(x) \int_{\sigma^{-1}(c)}^{x} h, \\
\mathcal{H}_{c}^{*} h(x) & :=\chi_{[c, \infty)}(x) \int_{x}^{\infty} h, \\
\mathcal{H}_{c, d}^{*} h(x) & :=\chi_{[c, d)}(x) \int_{x}^{\sigma(d)} h .
\end{aligned}
$$

Theorem 2.3. ([12], Theorems 2 and 3.) Let $1 \leq p<\infty, 0<r<\infty, 0<q<\infty$ and $\frac{1}{s}=\frac{1}{r}-\frac{1}{p}$ for $r<p$.

(a) For validity of the inequality (2.3) it is necessary and sufficient that the inequality

$$
\left(\int_{0}^{\infty} u(x)\left(\int_{x}^{\infty} w\right)^{\frac{r}{q}}\left(\int_{0}^{x} h\right)^{r} d x\right)^{\frac{1}{r}} \leq B_{0}\left(\int_{0}^{\infty} h^{p} v\right)^{\frac{1}{p}}, h \in \mathfrak{M}^{+},
$$

holds for all $h \in \mathfrak{M}^{+}$and the constant

$$
B_{1}:= \begin{cases}\sup _{t>0}\left(\int_{0}^{t} u\right)^{\frac{1}{r}}\left\|\mathcal{H}_{t}\right\|_{L_{v}^{p} \rightarrow L_{w}^{q}}, & p \leq r ; \\ \left(\int_{0}^{\infty} u(x)\left(\int_{0}^{x} u\right)^{\frac{s}{p}}\left\|\mathcal{H}_{\left[\sigma^{-1}(x), \sigma(x)\right]}\right\|_{L_{v}^{p} \rightarrow L_{w}^{q}}^{s} d x\right)^{\frac{1}{s}}, & r<p,\end{cases}
$$

is finite. Moreover, $C_{\mathcal{T}} \approx B_{0}+B_{1}$. 
(b) For validity of the inequality (2.4) it is necessary and sufficient that the inequality

$$
\left(\int_{0}^{\infty} u(x)\left(\int_{x}^{\sigma^{2}(x)} w\right)^{\frac{r}{q}}\left(\int_{\sigma^{2}(x)}^{\infty} h\right)^{r} d x\right)^{\frac{1}{r}} \leq \mathbf{B}_{0}\left(\int_{0}^{\infty} h^{p} v\right)^{\frac{1}{p}}, h \in \mathfrak{M}^{+},
$$

holds for all $h \in \mathfrak{M}^{+}$and the constant

$$
\mathbf{B}_{1}:= \begin{cases}\sup _{t>0}\left(\int_{0}^{t} u\right)^{\frac{1}{r}}\left\|\mathcal{H}_{t}^{*}\right\|_{L_{v}^{p} \rightarrow L_{w}^{q}}, & p \leq r ; \\ \left(\int_{0}^{\infty} u(x)\left(\int_{0}^{x} u\right)^{\frac{s}{p}}\left\|\mathcal{H}_{\left[\sigma^{-1}(x), \sigma(x)\right]}^{*}\right\|_{L_{v}^{p} \rightarrow L_{w}^{q}}^{s} d x\right)^{\frac{1}{s}}, & r<p\end{cases}
$$

is finite. Moreover, $C_{\mathcal{S}} \approx \mathbf{B}_{0}+\mathbf{B}_{1}$.

\section{MAin Results}

Suppose that $\mathfrak{S}: \mathfrak{M}^{+} \rightarrow \mathfrak{M}^{+}$is a positive monotone operator, that is for all $\left\{g_{n}\right\} \subset \mathfrak{M}^{+}, g \in \mathfrak{M}^{+}$such that $g_{n} \uparrow g$ it implies $\mathfrak{S}_{g_{n}} \uparrow \mathfrak{S} g$.

Lemma 3.1. Let $0<p, q<\infty ; u, v \in \mathfrak{M}^{+}, \varphi$ be admissible and let $\mathfrak{S}: \mathfrak{M}^{+} \rightarrow$ $\mathfrak{M}^{+}$be a positive monotone operator. Then the inequality

$$
\left(\int_{0}^{\infty}[\mathfrak{S} f(x)]^{q} u(x) d x\right)^{\frac{1}{q}} \leq C_{\mathfrak{S}}\left(\int_{0}^{\infty}[f(x)]^{p} v(x) d x\right)^{\frac{1}{p}}, f \in \Omega_{\varphi}
$$

is equivalent to

$$
\left(\int_{0}^{\infty}\left[\mathfrak{S}\left(B_{\varphi} h\right)^{\frac{1}{p}}(x)\right]^{q} u(x) d x\right)^{\frac{p}{q}} \leq C^{p} \int_{0}^{\infty} h(x) V(x) d x, h \in \mathfrak{M}^{+}
$$

where

and $C_{\mathfrak{S}} \approx C$.

$$
\begin{gathered}
B_{\varphi} h(x):=[\varphi(x)]^{p} \int_{0}^{\infty} \frac{h(z) d z}{[\varphi(x)]^{p}+[\varphi(z)]^{p}} \\
V(x):=\int_{0}^{\infty} \frac{[\varphi(z)]^{p} v(z) d z}{[\varphi(x)]^{p}+[\varphi(z)]^{p}}
\end{gathered}
$$

Proof. The proof follows by change $f^{p} \rightarrow f$ in (3.1), applying Lemma 2.1 and the equivalence

$$
\int_{0}^{[\varphi(x)]^{p}} d y \int_{y}^{\infty} \frac{h(z) d z}{z} \approx[\varphi(x)]^{p} \int_{0}^{\infty} \frac{h\left([\varphi(z)]^{p}\right) d\left([\varphi(z)]^{p}\right)}{[\varphi(x)]^{p}+[\varphi(z)]^{p}}
$$

Theorem 3.2. Let $0<p, q<\infty$ and $u, v, w \in \mathfrak{M}^{+}$. Then for the best constant $C_{A}$ of the inequality (1.1) we have

$$
C_{A} \approx A_{0}+A_{1}+\boldsymbol{A}_{0}+\boldsymbol{A}_{1}
$$


where the constants on the right-hand side are given or estimated by the following:

and

$$
A_{0}^{p}=\sup _{t>0} \frac{\left(\int_{0}^{t} u(x)\left(\int_{0}^{x} \varphi w\right)^{q} d x\right)^{\frac{p}{q}}}{[\varphi(t)]^{p} V(t)}, p \leq q
$$

$$
\begin{gathered}
A_{0}^{p} \approx\left(\int_{0}^{\infty}\left[(\varphi(t))^{p} V(t)\right]^{\frac{q}{q-p}} \times\right. \\
\left.\times\left(\int_{0}^{t} u(s)\left(\int_{0}^{s} \varphi w\right)^{q} d s\right)^{\frac{q}{p-q}} u(t)\left(\int_{0}^{t} \varphi w\right)^{q} d t\right)^{\frac{p-q}{q}}, q<p . \\
\boldsymbol{A}_{0}^{p}=\sup _{t>0} \frac{\left(\int_{\zeta^{2}(t)}^{\infty} u(x)\left(\int_{\zeta^{-2}(x)}^{x} w\right)^{q} d x\right)^{\frac{p}{q}}}{V(t)}, p \leq q
\end{gathered}
$$

and for $q<p$

$$
\boldsymbol{A}_{0}^{p} \approx\left(\int_{0}^{\infty}\left(\frac{\int_{t}^{\infty} u(s)\left(\int_{\zeta^{-2}(s)}^{s} w\right)^{q} d s}{V\left(\zeta^{-2}(t)\right)}\right)^{\frac{q}{p-q}} u(t)\left(\int_{\zeta^{-2}(t)}^{t} w\right)^{q} d t\right)^{\frac{p-q}{q}} .
$$

If $p \leq q$, then

$$
A_{1}=\sup _{t \in(0, \infty)}\left(\int_{t}^{\infty} u\right)^{\frac{1}{q}} \frac{\int_{0}^{t} \varphi w}{\varphi(t)[V(t)]^{\frac{1}{p}}}, 0<p \leq 1
$$

and

$$
A_{1} \approx \sup _{t \in(0, \infty)}\left(\int_{t}^{\infty} u\right)^{\frac{1}{q}}\left(\int_{0}^{t}\left(\frac{\int_{0}^{s} \varphi w}{[\varphi(s)]^{p} V(s)}\right)^{\frac{1}{p-1}} \varphi(s) w(s) d s\right)^{\frac{1}{p^{\prime}}}, p>1,
$$

where $p^{\prime}:=\frac{p}{p-1}$.

$$
\text { If } q<p \text {, then }
$$

$$
A_{1}=\left(\int_{0}^{\infty} u(x)\left(\int_{x}^{\infty} u\right)^{\frac{q}{p-q}}\left(\sup _{s \in\left(\zeta^{-1}(x), \zeta(x)\right)} \frac{\left(\int_{\zeta^{-1}(x)}^{s} \varphi w\right)^{p}}{[\varphi(s)]^{p} V(s)}\right)^{\frac{q}{p-q}} d x\right)^{\frac{p-q}{p q}}
$$

for $0<p \leq 1$ and

$$
\begin{aligned}
& A_{1} \approx\left(\int_{0}^{\infty} u(x)\left(\int_{x}^{\infty} u\right)^{\frac{q}{p-q}} \times\right. \\
& \left.\times\left(\int_{\zeta^{-1}(x)}^{\zeta(x)}\left(\frac{\int_{\zeta^{-1}(x)}^{t} \varphi w}{[\varphi(t)]^{p} V(t)}\right)^{\frac{1}{p-1}} \varphi(t) w(t) d t\right)^{\frac{q(p-1)}{p-q}} d x\right)^{\frac{p-q}{p q}}
\end{aligned}
$$

for $p>1$. 
When $p \leq q$

$$
\boldsymbol{A}_{1}=\sup _{t \in(0, \infty)}\left(\int_{t}^{\infty} u\right)^{\frac{1}{q}} \sup _{s \in(0, t)} \frac{\int_{s}^{t} w}{[V(s)]^{\frac{1}{p}}}, 0<p \leq 1
$$

and

$$
\boldsymbol{A}_{1} \approx \sup _{t \in(0, \infty)}\left(\int_{t}^{\infty} u\right)^{\frac{1}{q}}\left(\int_{0}^{t}\left(\frac{\int_{s}^{t} w}{V(s)}\right)^{\frac{1}{p-1}} w(s) d s\right)^{\frac{1}{p^{\prime}}}, p>1 .
$$

Let $q<p$. Then

$$
\boldsymbol{A}_{1}=\left(\int_{0}^{\infty} u(x)\left(\int_{x}^{\infty} u\right)^{\frac{q}{p-q}}\left(\sup _{s \in\left(\zeta^{-1}(x), \zeta(x)\right)} \frac{\left(\int_{s}^{\zeta(x)} w\right)^{p}}{V(s)}\right)^{\frac{q}{p-q}} d x\right)^{\frac{p-q}{p q}}
$$

if $0<p \leq 1$ and

$$
\begin{aligned}
& \boldsymbol{A}_{1} \approx\left(\int_{0}^{\infty} u(x)\left(\int_{x}^{\infty} u\right)^{\frac{q}{p-q}} \times\right. \\
& \left.\times\left(\int_{\zeta^{-1}(x)}^{\zeta(x)}\left(\frac{\int_{s}^{\zeta(x)} w}{V(s)}\right)^{\frac{1}{p-1}} w(s) d s\right)^{\frac{q(p-1)}{p-q}} d x\right)^{\frac{p-q}{p q}}
\end{aligned}
$$

for $p>1$.

Proof. By Lemma 3.1 and applying

$$
B_{\varphi} h(x) \approx[\varphi(x)]^{p} \int_{x}^{\infty} \frac{h}{[\varphi]^{p}}+\int_{0}^{x} h
$$

we see, that (1.1) is equivalent to the pair of inequalities:

$$
\left(\int_{0}^{\infty}\left[\int_{0}^{x}\left(\int_{y}^{\infty} h\right)^{\frac{1}{p}} \varphi(y) w(y) d y\right]^{q} u(x) d x\right)^{\frac{p}{q}} \lesssim C_{1}^{p} \int_{0}^{\infty} h[\varphi]^{p} V, h \in \mathfrak{M}^{+},
$$

and

$$
\left(\int_{0}^{\infty}\left[\int_{0}^{x}\left(\int_{0}^{y} h\right)^{\frac{1}{p}} w(y) d y\right]^{q} u(x) d x\right)^{\frac{p}{q}} \lesssim C_{2}^{p} \int_{0}^{\infty} h V, h \in \mathfrak{M}^{+},
$$

governing by Theorem 2.2, so we have

$$
C_{1} \approx A_{0}+A_{1}
$$

and

$$
C_{2} \approx \mathbf{A}_{0}+\mathbf{A}_{1}
$$

where $A_{0}$ is the least constant of the inequality

$$
\left(\int_{0}^{\infty} u(x)\left(\int_{0}^{x} \varphi w\right)^{q}\left(\int_{x}^{\infty} h\right)^{\frac{q}{p}} d x\right)^{\frac{p}{q}} \leq A_{0}^{p} \int_{0}^{\infty} h(z)[\varphi(z)]^{p} V(z) d z
$$


for all $h \in \mathfrak{M}^{+}$and $A_{1}$ is defined by

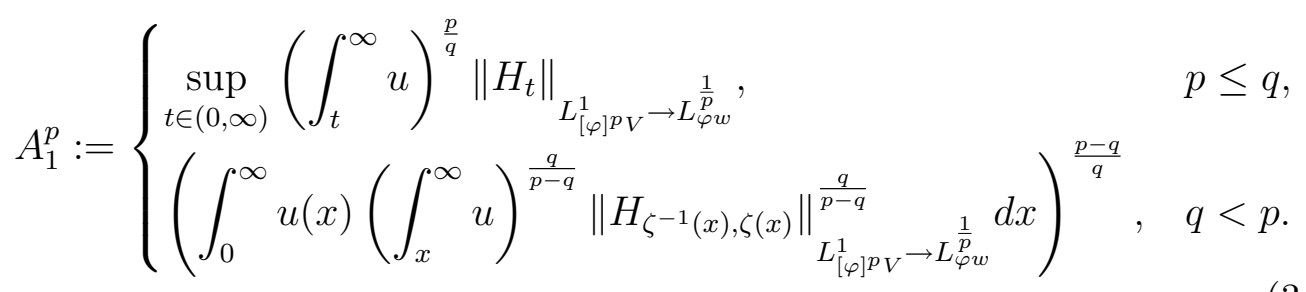

Analogously, $\mathbf{A}_{0}$ is the best possible constant in the inequality

$$
\left(\int_{0}^{\infty} u(x)\left(\int_{\zeta^{-2}(x)}^{x} w\right)^{q}\left(\int_{0}^{\zeta^{-2}(x)} h\right)^{\frac{q}{p}} d x\right)^{\frac{p}{q}} \leq \mathbf{A}_{0}^{p} \int_{0}^{\infty} h V, h \in \mathfrak{M}^{+}
$$

and $\mathbf{A}_{1}$ is determined from

$$
\mathbf{A}_{1}^{p}:= \begin{cases}\sup _{t \in(0, \infty)}\left(\int_{t}^{\infty} u\right)^{\frac{p}{q}}\left\|H_{t}^{*}\right\|_{L_{V}^{1} \rightarrow L_{w}^{\frac{1}{p}},} & p \leq q, \\ \left(\int_{0}^{\infty} u(x)\left(\int_{x}^{\infty} u\right)^{\frac{q}{p-q}}\left\|H_{\zeta^{-1}(x), \zeta(x)}^{*}\right\|_{L_{V}^{1} \rightarrow L_{w}^{\frac{1}{p}}}^{\frac{q}{p-q}} d x\right)^{\frac{p-q}{q}}, & q<p .\end{cases}
$$

If $k(x, y) \geq 0$ is a measurable kernel on $\mathbb{R}_{+} \times \mathbb{R}_{+}$and

$$
K f(x):=\int_{0}^{\infty} k(x, y) f(y) d y
$$

then by well known results ([10], Chapter XI, $\S 1.5$, Theorem 4, see also [7], Theorem 1.1)

$$
\|K\|_{L^{1} \rightarrow L^{\lambda}}=\sup _{t>0}\left(\int_{0}^{\infty}[k(x, t)]^{\lambda} d x\right)^{\frac{1}{\lambda}}, 1 \leq \lambda<\infty .
$$

Applying this result to the inequality (3.16) with $\lambda=\frac{q}{p}$ and

we find (3.3). If

$$
k(x, y)=\chi_{[x, \infty)}(y) \frac{[u(x)]^{\frac{p}{q}}\left(\int_{0}^{x} \varphi w\right)^{p}}{[\varphi(y)]^{p} V(y)}
$$

$$
\text { If }(x):=\rho(x) \int_{x}^{\infty} f(y) \varkappa(y) d y
$$

then by the dual version of ([15], Theorem 3.3) we have

$$
\|I\|_{L^{1} \rightarrow L^{\lambda}} \approx\left(\int_{0}^{\infty}\left[\operatorname{ess} \sup _{y>x} \varkappa(y)\right]^{\frac{\lambda}{1-\lambda}}\left(\int_{0}^{x} \rho\right)^{\frac{\lambda}{1-\lambda}} \rho(x) d x\right)^{\frac{1-\lambda}{\lambda}} .
$$

By this result with $\lambda=\frac{q}{p}$ applying to (3.16) we obtain (3.4) for $q<p$. Analogously, we find (3.5) and (3.6). Again, applying ([10], Chapter XI, $\S 1.5$, Theorem 4) and ([15], Theorem 3.3) we obtain

$$
\left\|H_{t}\right\|_{L_{[\varphi(z)]^{p} V(z)}^{1} \rightarrow L_{\varphi w}^{\frac{1}{p}}}=\sup _{s \in(0, t)} \frac{\left(\int_{0}^{s} \varphi w\right)^{p}}{[\varphi(s)]^{p} V(s)}, 0<p \leq 1
$$


and

$$
\left\|H_{t}\right\|_{L_{[\varphi(z)]^{1} V(z)}^{1} \rightarrow L_{\varphi w}^{\frac{1}{p}}} \approx\left(\int_{0}^{t}\left(\frac{\int_{0}^{s} \varphi w}{[\varphi(s)]^{p} V(s)}\right)^{\frac{1}{p-1}} \varphi(s) w(s) d s\right)^{p-1}, p>1,
$$

so that (3.17) brings (3.7) and (3.8). Similarly,

$$
\left\|H_{\zeta^{-1}(x), \zeta(x)}\right\|_{L_{[\varphi(z)]^{p} V(z)}^{1} \rightarrow L_{\varphi w}^{\frac{1}{p}}}=\sup _{s \in\left(\zeta^{-1}(x), \zeta(x)\right)} \frac{\left(\int_{\zeta^{-1}(x)}^{s} \varphi w\right)^{p}}{[\varphi(s)]^{p} V(s)}, 0<p \leq 1
$$

and

$\left\|H_{\zeta^{-1}(x), \zeta(x)}\right\|_{L_{[\varphi(z)]^{p} V(z)}^{1} \rightarrow L_{\varphi w}^{\frac{1}{p}}} \approx\left(\int_{\zeta^{-1}(x)}^{\zeta(x)}\left(\frac{\int_{\zeta^{-1}(x)}^{t} \varphi w}{[\varphi(t)]^{p} V(t)}\right)^{\frac{1}{p-1}} \varphi(t) w(t) d t\right)^{p-1}, p>1$.

Hence, (3.17) implies (3.9) and (3.10).

By the same way,

$$
\left\|H_{t}^{*}\right\|_{L_{V}^{1} \rightarrow L_{w}^{\frac{1}{p}}}=\sup _{s \in(0, t)} \frac{\left(\int_{s}^{t} w\right)^{p}}{V(s)}, 0<p \leq 1
$$

and

$$
\left\|H_{t}^{*}\right\|_{L_{V}^{1} \rightarrow L_{w}^{\frac{1}{p}}} \approx\left(\int_{0}^{t}\left(\frac{\int_{s}^{t} w}{V(s)}\right)^{\frac{1}{p-1}} w(s) d s\right)^{p-1}, p>1 .
$$

Now, (3.11) and (3.12) follow from (3.18). Moreover, we have

$$
\left\|H_{\zeta^{-1}(x), \zeta(x)}^{*}\right\|_{L_{V}^{1} \rightarrow L_{w}^{\frac{1}{p}}}=\sup _{s \in\left(\zeta^{-1}(x), \zeta(x)\right)} \frac{\left(\int_{s}^{\zeta(x)} w\right)^{p}}{V(s)}, 0<p \leq 1
$$

and

$$
\left\|H_{\zeta^{-1}(x), \zeta(x)}^{*}\right\|_{L_{V}^{1} \rightarrow L_{w}^{\frac{1}{p}}} \approx\left(\int_{\zeta^{-1}(x)}^{\zeta(x)}\left(\frac{\int_{s}^{\zeta(x)} w}{V(s)}\right)^{\frac{1}{p-1}} w(s) d s\right)^{p-1}, p>1 .
$$

Thus, from (3.18) we find (3.13) and (3.14) and the relations (3.3)-(3.14) are proved.

Remark 3.3. The diagonal case $\varphi(t)=t, u=v, 1<p=q<\infty$ was solved in [16].

Theorem 3.4. Let $0<p, q<\infty$ and $u, v, w \in \mathfrak{M}^{+}$. Then for the best constant $C_{B}$ of the inequality (1.2) we have

$$
C_{B} \approx \mathbf{B}_{\mathbf{0}}+\mathbf{B}_{\mathbf{1}}+B_{0}+B_{1},
$$


where the constants on the right-hand side are determined or sandwiched by the following relations.

$$
\mathbf{B}_{0}^{p}=\sup _{t>0}[V(t)]^{-1}\left(\int_{0}^{\sigma^{-2}(t)} u(x)\left(\int_{x}^{\sigma^{2}(x)} \varphi w\right)^{q} d x\right)^{\frac{p}{q}}, p \leq q
$$

and

$$
\begin{aligned}
& \mathbf{B}_{0}^{p} \approx\left(\int_{0}^{\infty}\left[V\left(\sigma^{2}(t)\right)\right]^{\frac{q}{q-p}} \times\right. \\
& \left.\times\left(\int_{0}^{t} u(x)\left(\int_{x}^{\sigma^{2}(x)} \varphi w\right)^{q} d x\right)^{\frac{q}{p-q}} u(t)\left(\int_{t}^{\sigma^{2}(t)} \varphi w\right)^{q} d t\right)^{\frac{p-q}{q}}
\end{aligned}
$$

for $q<p$.

$$
\begin{gathered}
B_{0}^{p}=\sup _{t>0}[V(t)]^{-1}\left(\int_{0}^{t} u(x)\left(\int_{x}^{\infty} w\right)^{q} d x\right)^{\frac{p}{q}}, p \leq q, \\
B_{0}^{p} \approx\left(\int_{0}^{\infty}[V(t)]^{\frac{q}{q-p}} \times\right. \\
\left.\times\left(\int_{t}^{\infty} u(x)\left(\int_{x}^{\infty} w\right)^{q} d x\right)^{\frac{q}{p-q}} u(t)\left(\int_{t}^{\infty} w\right)^{q} d t\right)^{\frac{p-q}{q}}, q<p .
\end{gathered}
$$

For $p \leq q$

$$
\begin{gathered}
\mathbf{B}_{1}=\sup _{t>0}\left(\int_{0}^{t} u\right)^{\frac{1}{q}} \sup _{s>t}\left[\varphi^{p}(s) V(s)\right]^{\frac{-1}{p}}\left(\int_{t}^{s} \varphi w\right), 0<p \leq 1 \\
\mathbf{B}_{1} \approx \sup _{t>0}\left(\int_{0}^{t} u\right)^{\frac{1}{q}}\left(\int_{t}^{\infty}\left(\frac{\int_{t}^{s} \varphi w}{\left[\varphi^{p}(s) V(s)\right]}\right)^{\frac{1}{p-1}} \varphi(s) w(s) d s\right)^{\frac{1}{p^{\prime}}}, p>1
\end{gathered}
$$

and for $q<p$

$$
\begin{aligned}
& \mathbf{B}_{1}=\left(\int_{0}^{\infty} u(x)\left(\int_{0}^{x} u\right)^{\frac{q}{p-q}} \times\right. \\
& \left.\times\left[\sup _{\sigma^{-1}(x)<s<\sigma(x)} \frac{\left(\int_{\sigma^{-1}(x)}^{s} \varphi w\right)^{p}}{\varphi^{p}(s) V(s)}\right]^{\frac{q}{p-q}} d x\right)^{\frac{p-q}{p q}}, 0<p \leq 1,
\end{aligned}
$$




$$
\begin{aligned}
& \mathbf{B}_{1} \approx\left(\int_{0}^{\infty} u(x)\left(\int_{0}^{x} u\right)^{\frac{q}{p-q}} \times\right. \\
& \left.\times\left(\int_{\sigma^{-1}(x)}^{\sigma(x)}\left(\frac{\int_{\sigma^{-1}(x)}^{s} \varphi w}{\varphi^{p}(s) V(s)}\right)^{\frac{1}{p-1}} \varphi(s) w(s) d s\right)^{\frac{(p-1) q}{p-q}} d x\right)^{\frac{p-q}{p q}},
\end{aligned}
$$

if $p>1$. For $p \leq q$

$$
\begin{gathered}
B_{1}=\sup _{t>0}\left(\int_{0}^{t} u\right)^{\frac{1}{q}} \sup _{s>t}[V(s)]^{\frac{-1}{p}}\left(\int_{s}^{\infty} w\right), 0<p \leq 1, \\
B_{1} \approx \sup _{t>0}\left(\int_{0}^{t} u\right)^{\frac{1}{q}}\left(\int_{t}^{\infty}\left(\frac{\int_{s}^{\infty} w}{V(s)}\right)^{\frac{1}{p-1}} w(s) d s\right)^{\frac{1}{p^{\prime}}}, p>1,
\end{gathered}
$$

and for $q<p$

$$
B_{1}=\left(\int_{0}^{\infty} u(x)\left(\int_{0}^{x} u\right)^{\frac{q}{p-q}}\left[\sup _{\sigma^{-1}(x)<s<\sigma(x)} \frac{\left(\int_{s}^{\sigma(x)} w\right)^{p}}{V(s)}\right]^{\frac{q}{p-q}} d x\right)^{\frac{p-q}{p q}}
$$

when $0<p \leq 1$ and

$$
\begin{aligned}
B_{1} & \approx\left(\int_{0}^{\infty} u(x)\left(\int_{0}^{x} u\right)^{\frac{q}{p-q}} \times\right. \\
& \left.\times\left(\int_{\sigma^{-1}(x)}^{\sigma(x)}\left(\frac{\int_{s}^{\sigma(x)} w}{V(s)}\right)^{\frac{1}{p-1}} w(s) d s\right)^{\frac{(p-1) q}{p-q}} d x\right)^{\frac{p-q}{p q}}, p>1 .
\end{aligned}
$$

Proof. By Lemma 3.1 and applying (3.15) we see, that (1.2) is equivalent to validity of the pair of inequalities

$$
\begin{gathered}
\left(\int_{0}^{\infty}\left[\int_{x}^{\infty}\left(\int_{y}^{\infty} h\right)^{\frac{1}{p}} \varphi(y) w(y) d y\right]^{q} u(x) d x\right)^{\frac{p}{q}} \leq \mathbf{C}_{1}^{p} \int_{0}^{\infty} h[\varphi]^{p} V, \\
\left(\int_{0}^{\infty}\left[\int_{x}^{\infty}\left(\int_{0}^{y} h\right)^{\frac{1}{p}} w(y) d y\right]^{q} u(x) d x\right)^{\frac{p}{q}} \leq \mathbf{C}_{2}^{p} \int_{0}^{\infty} h V
\end{gathered}
$$

for all $h \in \mathfrak{M}^{+}$, described by Theorem 2.3, so we have

$$
\mathrm{C}_{1} \approx \mathrm{B}_{\mathbf{0}}+\mathrm{B}_{1}
$$

and

$$
\mathbf{C}_{2} \approx B_{0}+B_{1},
$$


where $\mathbf{B}_{\mathbf{0}}$ is the least constant of the inequality

$$
\left(\int_{0}^{\infty} u(x)\left(\int_{x}^{\sigma^{2}(x)} \varphi w\right)^{q}\left(\int_{\sigma^{2}(x)}^{\infty} h\right)^{\frac{q}{p}} d x\right)^{\frac{p}{q}} \leq \mathbf{B}_{0}^{\mathbf{p}} \int_{0}^{\infty} h \varphi^{p} V, h \in \mathfrak{M}^{+} .
$$

and $\mathbf{B}_{\mathbf{1}}$ is defined by

$$
\mathbf{B}_{1}^{p}:= \begin{cases}\sup _{t>0}\left(\int_{0}^{t} u\right)^{\frac{p}{q}}\left\|\mathcal{H}_{t}^{*}\right\|_{L_{\varphi^{p} V}^{1} \rightarrow L_{\varphi w}^{\frac{1}{p}},} & p \leq q ; \\ \left(\int_{0}^{\infty} u(x)\left(\int_{0}^{x} u\right)^{\frac{q}{p-q}}\left\|\mathcal{H}_{\sigma^{-1}(x), \sigma(x)}^{*}\right\|_{\left.L_{\varphi^{p} V}^{1} \rightarrow L_{\varphi w}^{\frac{1}{p}} d x\right)^{\frac{q}{q}},}, q<p .\right.\end{cases}
$$

Analogously, $B_{0}$ is the least constant of the inequality

$$
\left(\int_{0}^{\infty} u(x)\left(\int_{x}^{\infty} w\right)^{q}\left(\int_{0}^{x} h\right)^{\frac{q}{p}} d x\right)^{\frac{p}{q}} \leq B_{0}^{p} \int_{0}^{\infty} h V, h \in \mathfrak{M}^{+}
$$

and $B_{1}$ is defined by

$$
B_{1}^{p}:= \begin{cases}\sup _{t>0}\left(\int_{0}^{t} u\right)^{\frac{p}{q}}\left\|\mathcal{H}_{t}\right\|_{L_{V}^{1} \rightarrow L_{w}^{\frac{1}{p}}} & p \leq q ; \\ \left(\int_{0}^{\infty} u(x)\left(\int_{0}^{x} u\right)^{\frac{q}{p-q}}\left\|\mathcal{H}_{\sigma^{-1}(x), \sigma(x)}\right\|_{L_{V}^{1} \rightarrow L_{w}^{\frac{1}{p}}}^{\frac{q}{p}} d x\right)^{\frac{1}{s}}, & q<p .\end{cases}
$$

Again, arguing as in the proof of Theorem 3.2 we obtain (3.19)-(3.30) and theorem is proved.

Acknowledgement. The research work of V.D. Stepanov was financially supported by the Russian Scientific Fund (Project 14-11-00443) and the research work of G.E. Shambilova was partially supported by the President grant for government support of the leading scientific schools of the Russian Federation No. 4479.2014.1.

\section{REFERENCES}

1. M. Ariño and B. Muckenhoupt, Maximal functions on classical Lorentz spaces and Hardy's inequality with weights for non-increasing functions, Trans. Amer. Math. Soc. 320 (1990), $727-735$.

2. C. Bennett and R. Sharpley, Interpolation of operators, Academic Press, Boston, 1988.

3. M.J. Carro, J.A. Raposo and J. Soria, Recent developments in the theory of Lorentz spaces and weighted inequalities, Memoirs Amer. Math. Soc. 877 (2007).

4. M.L. Goldman, H.P. Heinig and V.D. Stepanov, On the principle of duality in Lorentz spaces, Canad. J. Math. 48 (1996), no. 5, 959-979.

5. M.L. Goldman and M.V. Sorokina, Three-weighted Hardy-type inequalities on the cone of quasimonotone functions, Doklady Math. 71 (2005), no. 2, 209-213.

6. A. Gogatishvili and L. Pick, Discretization and antidiscretization of rearrangementinvariant norms, Publ. Mat. 47 (2003), 311-358.

7. A. Gogatishvili and V.D. Stepanov, Reduction theorems for weighted integral inequalities on the cone of monotone functions, Russian Math. Surveys, 68 (2013), no. 4, 597-664.

8. A. Gogatishvili, R. Mustafayev and L.-E. Persson, Some new iterated Hardy-type inequalities, J. Funct. Spaces Appl. 2012, Art. ID 734194, 30 pp. 
9. A. Gogatishvili, R. Mustafayev and L.-E. Persson, Some new iterated Hardy-type inequalities: the case $\theta=1$, J. Inequal. Appl. 2013, 2013:515.

10. L.V. Kantorovich and G.P. Akilov, Functional Analysis, Pergamon Press, Oxford, 1982.

11. L.-E. Persson, O.V. Popova and V.D. Stepanov Weighted Hardy-type inequalities on the cone of quasi-concave functions, Math. Inequal. Appl. 17, (2014), no 3, 879-898.

12. D.V. Prokhorov and V.D. Stepanov, On weighted Hardy inequalities in mixed norms, Proc. Steklov Inst. Math. 283 (2013), 149-164.

13. E. Sawyer, Boundedness of classical operators on classical Lorentz spaces, Studia Math. 96 (1990), 145-158.

14. G. Sinnamon, Embeddings of concave functions and duals of Lorentz spaces, Publ. Mat. 46 (2002), 489-515.

15. G. Sinnamon and V.D. Stepanov, The weighted Hardy inequality: new proofs and the case $p=1$. J. London Math. Soc. 54 (1996), 89-101.

16. V.D. Stepanov, Integral operators on the cone of monotone functions, J. London Math. Soc. 48 (1993), 465-487.

${ }^{1}$ Department of Engineering Sciences and Mathematics, Lulea University of Technology, SE-97187 Lulea, Sweden, and Narvik University College, PO Box 385, NO 8505, NARVIK, NORWAY.

E-mail address: Lars-Erik.Persson@ltu.se

2 Department of Mathematical Analysis and Function Theory, Peoples' FriendShip University of Russia, Miklukho-Maklay 6, 117198 Moscow, Russia.

E-mail address: shambilova@mail.ru

${ }^{3}$ Department of Mathematical Analysis and Function Theory, Peoples' FriendShip University of Russia, Miklukho-Maklay 6, 117198 Moscow, Russia;

Steklov Mathematical Institute, Russian Academy of Sciences, Gubkina 8, 119991 Moscow, Russia.

E-mail address: stepanov@mi.ras.ru 